\title{
L'éducation relative à l'environnement dans la Réserve de biosphère du mont Saint-Hilaire : observations et tensions
}

Marc-André Guertin, Geneviève Poirier-Ghys, Maureen G. Reed, Jérôme Dupras et Geneviève Rajotte Sauriol

\section{(2) OpenEdition}

\section{Journals}

Édition électronique

URL : https://journals.openedition.org/ere/3810

DOI : $10.4000 /$ ere.3810

ISSN : 2561-2271

Éditeur

Centr'ERE

Référence électronique

Marc-André Guertin, Geneviève Poirier-Ghys, Maureen G. Reed, Jérôme Dupras et Geneviève

Rajotte Sauriol, «L'éducation relative à l'environnement dans la Réserve de biosphère du mont SaintHilaire : observations et tensions », Éducation relative à l'environnement [En ligne], Volume 15 - 1 | 2019, mis en ligne le 20 décembre 2019, consulté le 16 octobre 2021. URL : http://journals.openedition.org/ ere/3810 ; DOI : https://doi.org/10.4000/ere.3810

Ce document a été généré automatiquement le 16 octobre 2021.

La revue Éducation relative à l'environnement est mise à disposition selon les termes de la Licence Creative Commons Attribution - Pas d'Utilisation Commerciale 4.0 International. 


\title{
L'éducation relative à
}

\section{l'environnement dans la Réserve de biosphère du mont Saint-Hilaire : observations et tensions}

\author{
Marc-André Guertin, Geneviève Poirier-Ghys, Maureen G. Reed, Jérôme \\ Dupras et Geneviève Rajotte Sauriol
}

1 Cet article s'attarde aux pratiques éducatives suggérées par l'Organisation des Nations Unies pour l'éducation, la science et la culture (UNESCO) au sein des aires protégées. Au début des années 1970, sous l'égide de l'UNESCO, des initiatives institutionnelles majeures concernant le réseau de relations personne-société-environnement ont vu le jour, notamment la Déclaration de Tbilissi (UNESCO-PNUE, 1977), ainsi que le Programme de l'Homme et la Biosphère de l'UNESCO (en anglais, Man and Biosphere, à l'origine de l'acronyme MAB ; UNESCO, 2017).

2 Le Programme MAB vise à établir une base scientifique pour améliorer les rapports des populations à l'environnement au niveau mondial (UNESCO, 2019a). Après presque 50 ans d'existence, l'expression la plus tangible du Programme MAB est un réseau de plus de 701 réserves de biosphère réparties dans 124 pays (UNESCO, 2019b). Les réserves de biosphère englobent des aires protégées et souvent de plus vastes territoires afin d'encourager les communautés locales à conserver la biodiversité, à promouvoir le développement durable, ainsi qu'à renforcer leurs capacités logistiques notamment par la recherche et l'éducation (UNESCO, 1996).

C'est à ce dernier point que nous nous attardons dans cet article. Afin de mieux saisir comment s'exprime la dimension éducative du Réseau mondial des réserves de biosphère, nous avons mené une étude de cas visant à examiner de façon critique les pratiques en ce sens au sein de l'une de ces réserves de biosphère située au mont SaintHilaire, en banlieue de Montréal au Québec ${ }^{1}$. Celle-ci a d'abord été réalisée dans le cadre $\mathrm{du} 3^{\mathrm{e}}$ examen périodique de la Réserve de biosphère du mont Saint-Hilaire (RBMSH) (Gagnon, 2012). L'article 4 du cadre statutaire qui régit le Réseau mondial des réserves 
de biosphère prévoit qu'un bilan soit effectué tous les dix ans sur les actions et les résultats obtenus (UNESCO, 1996, 2017a). Bien que cet exercice puisse avoir une portée normative pouvant mener jusqu'à la radiation du Réseau mondial des réserves de biosphère ${ }^{2}$, l'examen périodique est aussi abordé, comme le suggèrent Reed et Egunyu (2013) de manière formative, systématique et réflexive afin de partager les apprentissages qui en découlent.

Cette étude de cas poursuit trois objectifs. Premièrement, analyser les orientations et actions suggérées par le cadre statutaire et les plans d'action du Programme MAB pour les réserves de biosphère en matière d'éducation. Deuxièmement, recenser les initiatives d'éducation relative à l'environnement (ERE) déployées dans la RBMSH durant les 10 dernières années. Troisièmement, jeter un regard critique sur les actions et orientations suggérées par le Programme MAB, ainsi que les pratiques d'ERE déployées dans la RBMSH.

5 Pour ce faire, l'article présente d'abord un bref portrait de la stratégie, du cadre statutaire et des plans d'action relatifs aux pratiques éducatives prescrites par le Programme MAB et l'UNESCO. Suivra un aperçu des activités d'ERE recensées sur le territoire de la RBMSH. Finalement, la dernière section propose une analyse critique de ces observations afin d'alimenter une réflexion relative à certaines tensions associées aux pratiques en ERE dans la RBMSH, ainsi que dans le Réseau mondial des réserves de biosphère.

\section{Vision de l'éducation dans le Réseau mondial des réserves de biosphère}

6 L'analyse présentée dans cette section est le fruit de plusieurs années d'observation participante à différentes échelles dans le Réseau mondial des réserves de biosphère, à la fois comme praticiens dans certaines réserves de biosphère québécoises, comme membre du Comité MAB de la Commission canadienne pour l'UNESCO et comme participants dans des forums nationaux et internationaux associés au Programme MAB et à l'UNESCO. Ces observations ont été complétées par une analyse thématique par mots clés dans les documents statutaires et normatifs, ainsi qu'une recension d'écrits dans les bases de données du Education Resources Information Center (ERIC) et d'Elsevier's abstract and citation database (SCOPUS) en septembre 2017.

7 Le Programme MAB encadre le Réseau mondial des réserves de biosphère à l'aide de stratégies. C'est de la première, appelée la Stratégie de Séville, que découle un cadre statutaire déterminant les règles de fonctionnement du Réseau mondial ainsi que des directives auxquelles doivent se conformer les réserves de biosphère. Par ailleurs, deux plans d'action et une nouvelle stratégie ont jalonné les deux dernières décennies, en suggérant des interventions à réaliser à différentes échelles par le Réseau mondial des réserves de biosphère. Cette section dresse un portrait sommaire des activités éducatives suggérées dans ces écrits.

8 La Stratégie de Séville (élaborée en Espagne lors du Congrès mondial des réserves de biosphère en 1996, ci-après nommée la Stratégie) clarifie le concept de réserve de biosphère en proposant une vision, de grands objectifs, des indicateurs de performance, ainsi qu'un cadre statutaire devant servir à baliser la mise en œuvre du concept, tout en tenant compte de la diversité des contextes culturels et organisationnels des pays 
membres. Relativement à l'éducation, l'UNESCO $(1996$, p. 5) précise le rôle de ces réserves :

[.... 熙 les réserves de biosphère constituent des sites privilégiés pour la recherche, la surveillance à long terme, la formation, l'éducation et la sensibilisation du public tout en permettant d'associer pleinement les communautés locales à la conservation et à l'utilisation durable des ressources. ${ }^{3}$

9 Fait à noter, l'éducation, la formation et la sensibilisation sont souvent présentées comme des pratiques complémentaires, bien qu'elles ne soient pas distinctement explicitées.

10 La vision de la Stratégie fait aussi référence à l'éducation, à l'aide de deux orientations qui précisent la nécessité de :

4. Renforcer la recherche scientifique, la surveillance continue, la formation et l'éducation dans les réserves de biosphère, en raison de la nécessité d'avoir une base solide dans le domaine des sciences naturelles et sociales, afin de mieux promouvoir la conservation et l'utilisation durable des ressources naturelles dans ces sites. (p.9)

10. Investir dans le futur: les réserves de biosphère devraient être utilisées pour approfondir notre connaissance des relations de l'humanité avec le milieu naturel, grâce à des programmes de vulgarisation, d'information, et d'éducation dans une perspective intergénérationnelle à long terme [sic]. (p.9)

11 L'« éducation à l'environnement » est ainsi évoquée de façon parcimonieuse dans la Stratégie, et ce, dans une perspective d'éducation au sujet de l'environnement. De plus, sans nommer l'éducation au développement durable (EDD) proprement dite, le concept de durabilité est omniprésent dans les orientations associées à la vision ainsi que dans les objectifs de la Stratégie ${ }^{4}$.

12 Les objectifs de la Stratégie permettent de favoriser une compréhension plus approfondie des pratiques éducatives suggérées. L'objectif 3.1 suggère d'améliorer les connaissances sur les interactions entre les humains et la biosphère en ayant recours notamment à des universités et à d'autres instituts d'enseignement supérieur et de recherche. L'objectif 3.3 propose quant à lui d'améliorer l'éducation, la sensibilisation $\mathrm{du}$ public ainsi que la participation citoyenne, cette fois par l'entremise d'une large gamme de pratiques allant de la révision des programmes scolaires à la promotion d'activités en nature dans les réserves. Finalement, l'objectif 3.4 spécifie la nature des activités de formation suggérées pour des spécialistes, gestionnaires et décideurs locaux portant sur des thèmes comme la surveillance continue des écosystèmes, l'analyse et l'étude des conditions socioculturelles, la résolution des conflits et la gestion des ressources de manière coopérative. Le cadre statutaire reprend l'essentiel des pratiques éducatives évoquées dans la Stratégie, sans réellement permettre d'en approfondir la portée. Il ne fait qu'évoquer le caractère essentiel de l'éducation afin d'être reconnu à titre de réserve de biosphère (UNESCO, 1996).

13 En 2008, un plan d'action est proposé pour orienter plus spécifiquement les actions du Réseau mondial des réserves de biosphère pour la période 2008-2013 (UNESCO, 2008). Ce plan, intitulé le Plan d'action de Madrid (élaboré en Espagne lors du Congrès mondial des réserves de biosphère en 2008), marque un changement important en ce qui a trait aux prescriptions éducatives du Programme MAB. Les réserves de biosphère sont maintenant invitées à devenir des sites d'apprentissage pour le développement durable. La mission évoquée dans le plan fait référence aux défis socioécologiques de conservation et d'utilisation durable de la biodiversité, d'adaptation au changement 
climatique et d'atténuation de ses effets, ainsi que pour contribuer au bien-être socioéconomique et culturel des communautés humaines. La mission du plan spécifie qu'il faut développer «(...) les capacités globales de gestion de systèmes socioécologiques complexes, notamment en développant le dialogue entre scientifiques et politiques, l'éducation environnementale et l'utilisation du multimédia pour toucher un public plus large» (p.9). Fait à noter, l'ERE est évoquée uniquement dans cet élément de la mission. Par la suite, lorsque l'éducation est mentionnée dans le plan, on fait surtout référence à l'éducation au développement durable ou encore au développement des capacités (p.25). La portée de ce plan d'action vise le Réseau mondial des réserves de biosphère ainsi que les répondants nationaux du Programme MAB. Il offre peu de précisions en ce qui a trait aux activités éducatives dans les réserves de biosphère à l'échelle locale.

La nouvelle stratégie du Programme MAB et le plan d'action de Lima visant la période 2016-2025 (adopté au Pérou lors du Congrès mondial des réserves de biosphère en 2016) viennent aussi apporter des changements importants, situant toutes les actions du Programme MAB dans une perspective de «sciences de la durabilité » (UNESCO, 2016). Cela s'applique aussi aux pratiques éducatives. L'ERE est complètement évacuée de ce plan au profit de l'EDD. Pourtant, la vision du plan réaffirme la nécessité de repenser notre rapport à la biosphère et de former des écocitoyens.

Notre vision est celle d'un monde où les hommes ont conscience de leur avenir commun et de leur interaction avec notre planète et agissent de façon collective et responsable pour construire des sociétés prospères en harmonie avec la biosphère.

Au terme d'une décennie consacrée à la promotion de l'EDD (Buckler \& Creech, 2014), l'éducation devient une stratégie transversale qui recoupe plusieurs domaines d'action stratégique pour soutenir le Réseau mondial des réserves de biosphère. Les pratiques éducatives doivent aussi tenir compte des objectifs du développement durable des Nations Unies (ODD) (Nations Unies, 2016). Les pratiques éducatives visent la formation d'acteurs spécifiques, notamment les organisations privées. Dans ce plan d'action, l'éducation constitue une stratégie de développement des capacités, c'est-à-dire une stratégie pour renforcer les habiletés à réaliser des tâches et notamment les ODD au sein du Réseau mondial des réserves de biosphère (FAO, 2010 ; Nations Unies, 2008).

Comme le suggèrent Sauvé et coll. (2003), les propositions internationales peuvent servir de balises pour guider et légitimer les choix éducationnels dans les pays, pourvu qu'elles soient adaptées dans une perspective critique. Voyons maintenant comment les acteurs de la RBMSH naviguent entre certaines tensions associées à leurs pratiques et les balises offertes par la Stratégie de Séville ainsi que les plans d'action de Madrid et de Lima. Ces documents étant ceux qui étaient en vigueur durant la période visée par l'examen périodique.

\section{RBMSH : survol des pratiques en éducation relative à l'environnement}

En 1978, la Réserve de biosphère du mont Saint-Hilaire (RBMSH) est la première à être désignée par l'UNESCO au Canada (Centre de la Nature, 2017). La majeure partie de l'aire protégée centrale de la réserve appartient à l'Université McGill, une institution d'enseignement supérieur vouée à la recherche et à l'éducation. La RBMSH est actuellement coordonnée par le Centre de la Nature, une organisation non 
gouvernementale (ONG) qui possède plus de 40 ans d'expérience en ERE et qui a développé au fil du temps une grande diversité d'activités éducatives en partenariat avec des municipalités, des ONG et des institutions d'enseignement présentes sur le territoire de la réserve. Plus de 300000 personnes fréquentent annuellement l'aire protégée centrale de la RBMSH, située sur cette colline montérégienne, pour y pratiquer des activités de plein air et d'interprétation de la nature. De plus, 44000 personnes habitent les huit municipalités de la RBMSH. Contrairement à bien des réserves de biosphère au Canada et au Québec, elle dispose de moyens financiers, d'employés et de bénévoles lui permettant le déploiement d'une diversité de pratiques en ERE (Centre de la Nature, 2017).

Les éléments d'information retrouvés dans cette section sont issus des archives du Centre de la Nature ainsi que de divers documents produits par des partenaires locaux et visent à recenser et catégoriser les activités d'ERE qui se déroulent en lien avec la RBMSH.

19 Sur le territoire de la RBMSH, on dénombre trois commissions scolaires et treize établissements d'enseignement : dix de niveau primaire, deux de niveau secondaire et un de niveau universitaire. Parmi les écoles primaires, cinq écoles mènent des projets éducatifs associés au programme des écoles vertes Brundtland (EVB-CSQ) qui vise à éduquer en fonction de valeurs écologiques, pacifiques, solidaires et démocratiques (EVB-CSQ, 2017).

20 De plus, environ 400 étudiants universitaires reçoivent de la formation spécialisée dans divers domaines associés aux sciences naturelles et à la conservation. On y offre notamment des cours de géographie physique, de limnologie, d'écologie et de botanique dans la RBMSH (McGill, 2017).

21 On laisse à la discrétion d'un établissement scolaire d'établir un projet éducatif au Québec (MEES, 2017). Aucun établissement scolaire du territoire n'a de projet éducatif faisant directement référence à la réserve de biosphère. Certains établissements y font référence, mais les activités éducatives relatives à la réserve de biosphère dans ces établissements sont issues d'initiatives d'enseignants ou d'ONG comme le Centre de la Nature (Centr'ERE, 2018 ; Centre de la Nature, 2017).

Pour cette étude de cas, une trentaine d'activités et d'outils pédagogiques, offerts sur le territoire ont été recensés ${ }^{5}$ (Tableau 1). Elles ont été classées par types de participants : des élèves et étudiants, des visiteurs, des citoyens, des élus et des professionnels municipaux. La majeure partie d'entre elles se déroulent à l'extérieur des établissements d'enseignement et rejoignent un vaste éventail de participants adultes. 
Tableau 1 : Activités et outils pédagogiques en ERE recensés dans la RBMSH

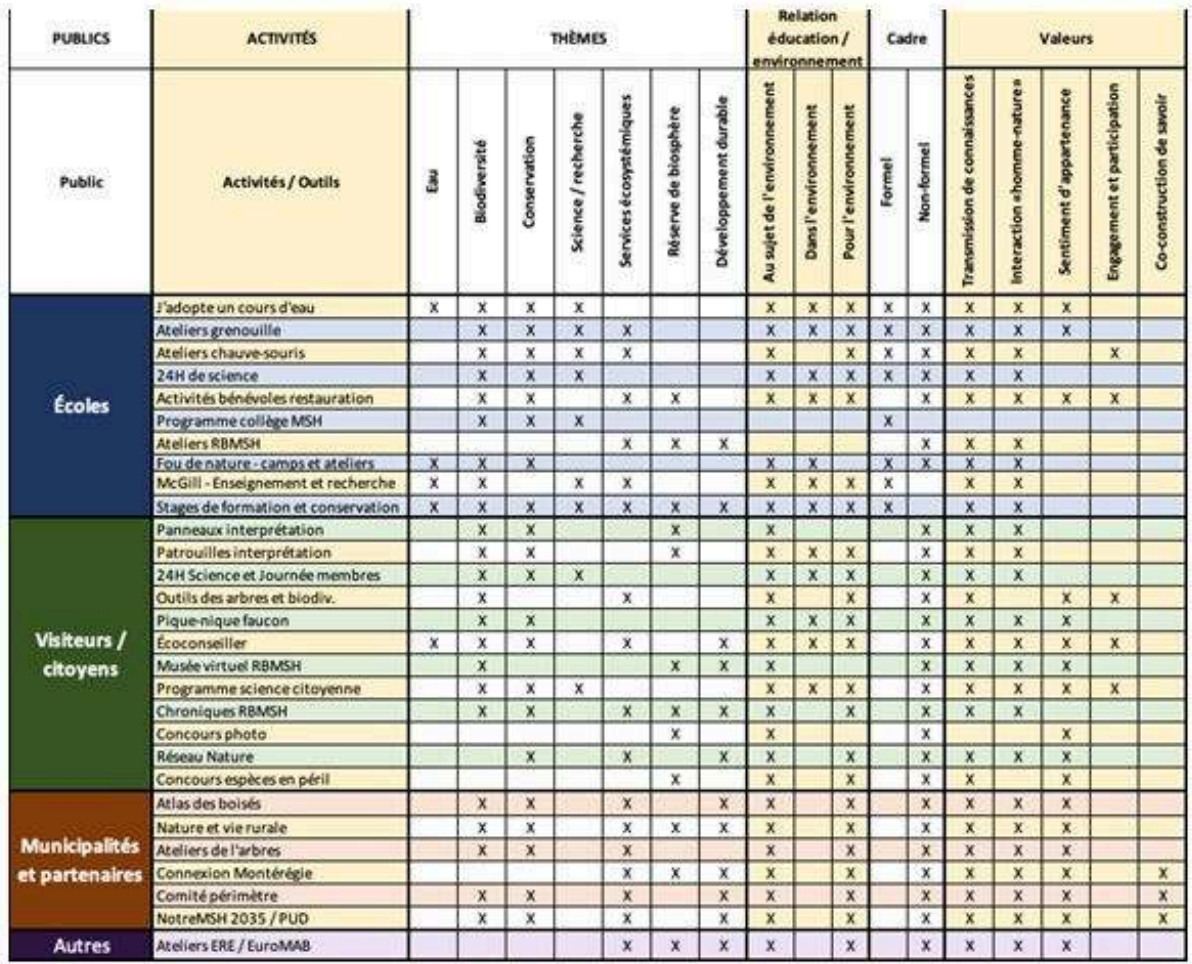

23 Les activités abordent principalement les thèmes de la biodiversité, de sa conservation et de l'importance des services écosystémiques. Dans une plus faible proportion, elles se penchent sur les concepts de réserve de biosphère et de développement durable, sans oublier les thèmes socioécologiques comme l'eau, la pollution, la recherche, les sciences ainsi que l'histoire naturelle et culturelle. À titre d'exemple, les pratiques prennent la forme de chroniques dans les journaux, d'ateliers avec les établissements scolaires, de programmes de science citoyenne (ex.: inventaires d'oiseaux, d'amphibiens ou de reptiles), de rencontre avec des écoconseillers ou d'outils interactifs sur Internet comme des cartes interactives ou encore un musée virtuel en ligne pour découvrir la RBMSH (Centre de la Nature, 2007).

Les pratiques éducatives peuvent aussi être triées par intention pédagogique. Bien qu'elles permettent toutes de transmettre des connaissances et de réfléchir au rapport humain-nature, certaines permettent aussi de cultiver un sentiment d'appartenance au territoire ou, dans une plus faible mesure, de favoriser l'engagement et l'utilisation de scénarios de résolution de problème. Les activités recensées comportent souvent des intentions pédagogiques multiples visant à la fois à cultiver un sentiment d'appartenance et à s'engager dans des projets ou actions de conservation en plus de rassembler plusieurs participants potentiels (ex.: étudiants, adultes, élus, fonctionnaires).

Ce survol a permis de formuler un certain nombre d'observations en ce qui a trait à l'ERE dans la RMBSH et, de façon plus générale, à l'éducation au sein d'une réserve de biosphère. 


\section{L'éducation dans la RBMSH en cinq observations}

La revue des écrits des réserves de biosphère concernant l'éducation a permis de comprendre la portée des guides internationaux qui encadrent ces pratiques ainsi que celles de la RBMSH dans un contexte québécois. Cette section vise à effectuer une lecture transversale des activités d'ERE réalisées dans la RBMSH et nous permet de formuler cinq observations en plus d'identifier certaines tensions.

\section{Des pratiques éducatives « au sujet de » et « pour » l'environnement}

Dans le recensement des activités éducatives de la RBMSH, on remarque une diversité de conceptions de l'environnement, de l'environnement-nature à l'environnementproblème ou encore, à l'environnement-ressource (Sauvé, 1997, p. 14). Il en est de même dans les écrits du Programme MAB où l'on remarque à travers le temps, un délestage de la conception de l'environnement-biosphère (Homme et biosphère) pour une conception de l'environnement-problème et de l'environnement-ressource (Sauvé, 1997b).

À partir des écrits du Programme MAB analysés et du recensement des pratiques dans la RBMSH, une perspective environnementale de l'ERE semble prévaloir : on remarque en effet que les pratiques éducatives suggérées tentent de trouver réponse aux problématiques de dégradation de l'environnement (Sauvé, 1997, p.22). Les perspectives sociales et pédagogiques de l'ERE sont moins développées dans les écrits du Programme MAB. Il en est de même dans les activités recensées au sein de la RBMSH.

La diversité des activités recensées témoigne aussi d'une pluralité de conceptions de la relation éducation-environnement (Sauvé, 1997b). Le recensement met en évidence des activités éducatives qui se pratiquent surtout «au sujet» de l'environnement et du concept de réserve de biosphère ainsi que "pour» l'environnement (Figure 1). Par ailleurs, on remarque dans une moindre mesure, des pratiques « dans » la nature ${ }^{6}$. Ces activités reflètent, à bien des égards, les pratiques recensées par Godmaire et coll. (2013) dans d'autres réserves de biosphère au Canada et pourraient servir de base à une étude comparative à l'échelle canadienne ou internationale.

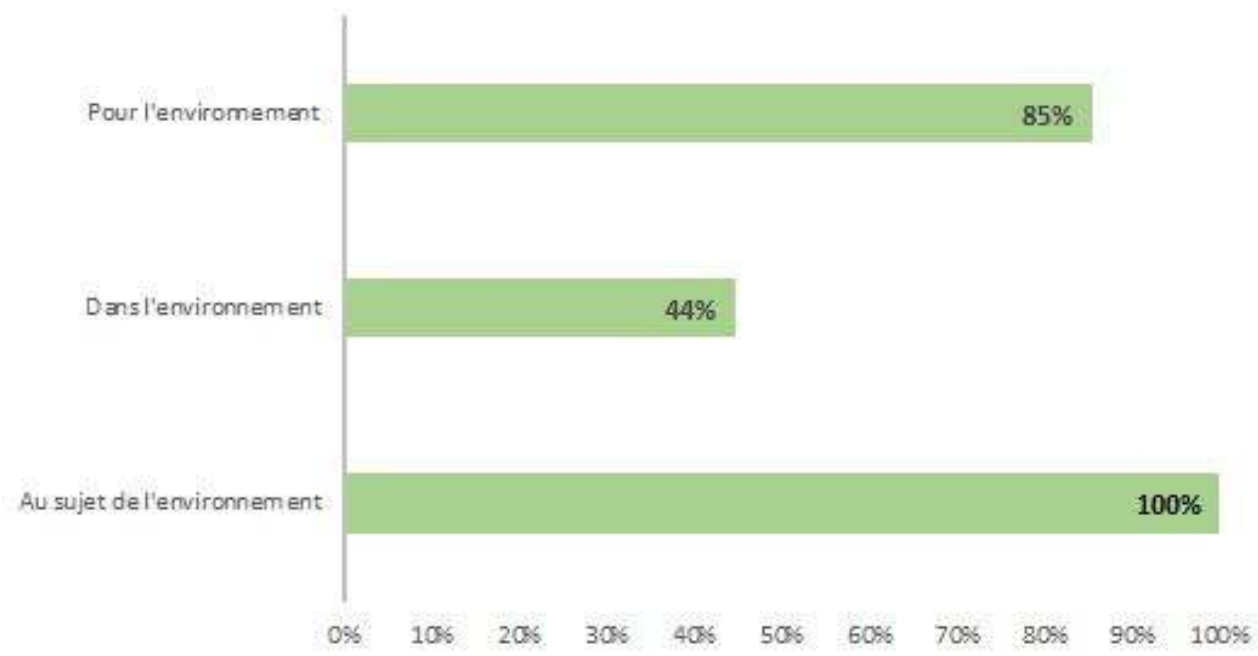


Figure 1 : Des pratiques éducatives « pour », « dans » et « au sujet de » l'environnement

\section{Les pratiques éducatives de la RBMSH dans un cadre essentiellement non formel}

Bien que la Stratégie de Séville et les plans d'action associés au Programme MAB suggèrent d'intégrer le concept de réserve de biosphère aux programmes scolaires (UNESCO, 1996), on remarque peu d'exemples à cet effet sur le territoire de la RBMSH. Rappelons qu'au Québec, l'éducation relève de la compétence provinciale et que l'ERE est associée à un domaine général de formation (MEQ, 2010). L'ERE est ainsi sousdéveloppée et sans ancrage territorial (Centr'ERE et coll., 2017). Les écoles ont la liberté de déployer des projets d'établissement ou des activités pédagogiques qui auraient pu octroyer une place au concept de réserve de biosphère ; toutefois, cette étude de cas n'a pas permis d'identifier des initiatives probantes à cet effet. Sur les 30 pratiques recensées, 10 sont offertes aux élèves et aux étudiants des institutions d'enseignement présentes au sein de la RBMSH (du niveau primaire au niveau universitaire) et une vingtaine sont offertes dans un cadre non formel à des participants adultes ou à des acteurs municipaux.

Dans la RBMSH, plusieurs activités et outils ont été développés afin de répondre aux enjeux environnementaux ainsi que pour promouvoir indirectement la réserve de biosphère. Comme l'affirment certains praticiens de la RBMSH, mieux vaut agir comme une réserve de biosphère plutôt que d'en discuter (Raufflet, et coll., 2010). Force est de constater que l'ERE se déploie surtout dans un cadre non formel (Figure 2). Plusieurs motifs peuvent être suggérés pour expliquer cette tension, notamment les modestes ressources dont dispose la RBMSH, et les réserves de biosphère en général au Canada, pour rejoindre un nombre aussi important d'élèves, d'étudiants et d'établissements scolaires (Bridgewater, 2016; Francis, 2004; Price, 2017), ainsi que la place de l'ERE dans le cursus scolaire québécois (Centr'ERE. 2018).

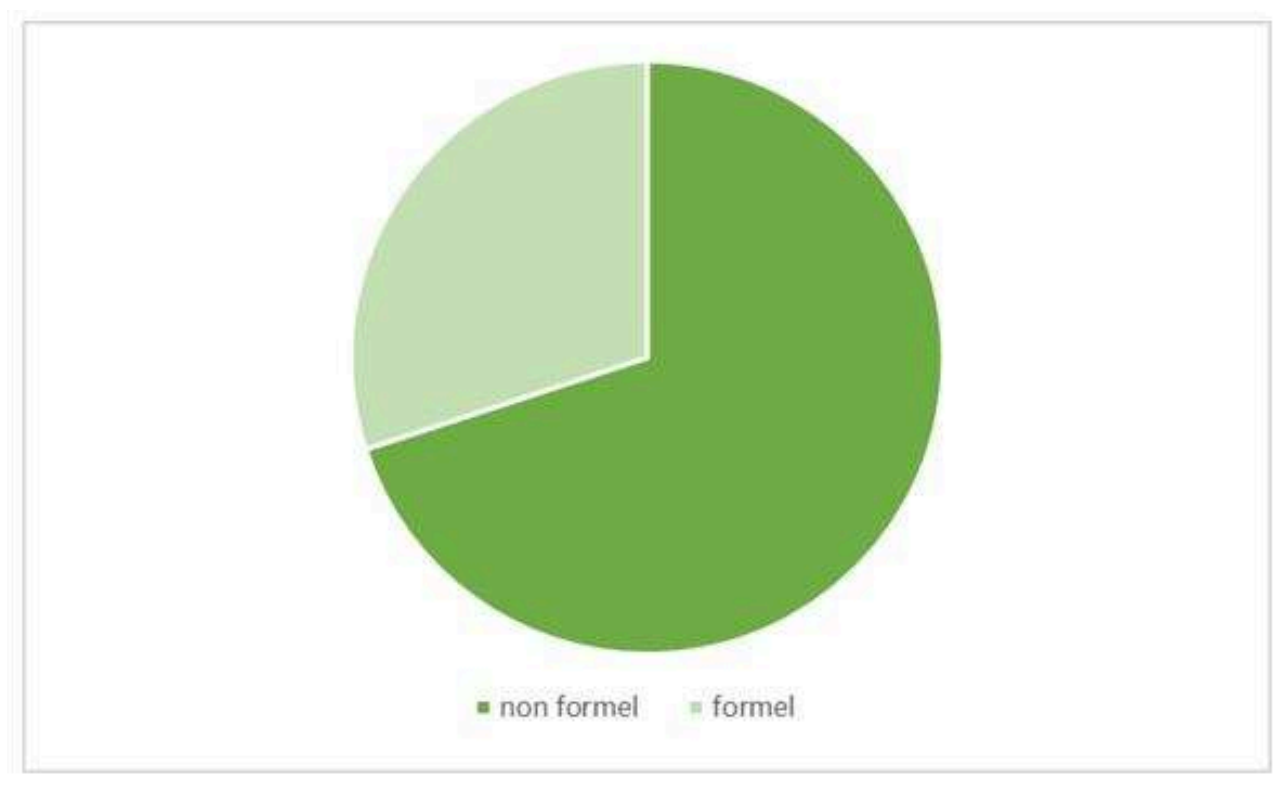


Figure 2 : Le contexte des pratiques éducatives de la RBMSH : prévalence du cadre non formel.

\section{Une axiologie des pratiques éducatives}

Comme en témoignent les deux observations précédentes, les pratiques éducatives proposées par le Programme MAB et celles recensées dans la RBMSH reposent sur diverses conceptions de l'environnement et des relations entre environnement et éducation. De plus, elles se déploient surtout dans un cadre non formel au sein de la RBMSH. Peu de références sont établies avec les valeurs que ces pratiques soutiennent ou qu'elles tentent de promouvoir. Elles comportent souvent plus d'une intention pédagogique. L'importance des problématiques environnementales de la RBMSH comme la destruction des milieux naturels et l'introduction de multiples espèces exotiques envahissantes offre une légitimité implicite aux pratiques éducatives utilisées. Bien qu'elles visent principalement à transmettre des connaissances relatives à la nature et à susciter un sentiment d'appartenance aux milieux naturels, la perspective environnementale dominante de l'ERE (et le manque de prise en compte des dimensions sociales des réalités écologiques) ne facilite pas ici la réflexion critique sur les causes sous-jacentes à ces problématiques (Figure 3). Par ailleurs, les pratiques visant la participation citoyenne ou la coconstruction des savoirs mènent à un questionnement général à l'égard de l'environnement; elles incitent aussi à la participation à des activités de conservation ou de restauration à l'échelle locale. Mais elles ne facilitent pas la réflexion éthique plus large et l'identification de conflits de valeurs qui sous-tendent les questions environnementales, de nature socio-écologiques.

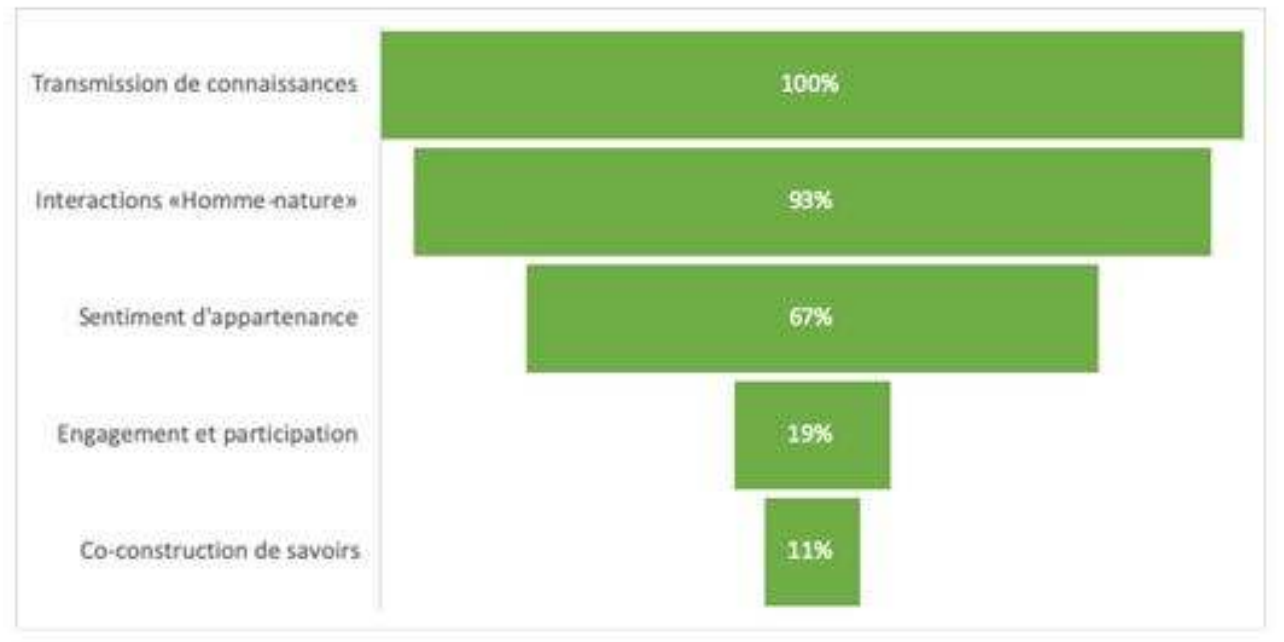

Figure 3 : Axiologie des pratiques éducatives

Par ailleurs, l'EDD est souvent invoquée dans les écrits du Programme MAB et très peu dans les pratiques éducatives recensées dans la RBMSH. Comme le suggèrent Scoullos et coll. (2013), la culture de l'EDD n'a pas vraiment pénétré les réserves de biosphère ayant une tradition d'ERE, comme c'est le cas dans la RBMSH. Il semble que les acteurs de la RBMSH aient dénoué cette tension en adoptant la posture de «faire sans » l'EDD, pour reprendre l'expression de Girault et Sauvé (2008). Il serait peut-être pertinent que le Programme MAB adopte une posture moins normative en ce qui a trait aux pratiques de la conservation (Sandbrook, et coll., 2013) et aux pratiques éducatives (Brianso et 
Girault, 2014 ; Girault et Sauvé, 2008) de manière à aborder les dimensions éthiques et politiques associées à ces pratiques. Cela permettrait de mieux rendre compte de la diversité des relations humain-société-environnement et des rapports entre éducation et environnement.

\section{Pour améliorer les pratiques éducatives : un modèle-cadre en ERE} ont participé à des travaux de recherche et de formation portant sur l'ERE et l'apprentissage collectif (Godmaire, Reed et Guertin, 2013 ; Godmaire, Reed, Potvin et Réserves de biosphère canadiennes, 2013 ; Reed et Abernethy, 2018; Reed, Godmaire, Abernethy et Guertin, 2014 ; Reed et Massie, 2013). Ces travaux ont permis d'identifier de «bonnes pratiques» dans divers domaines comme l'aménagement du territoire, le tourisme et l'éducation. Il s'agit bien ici du regard d'acteurs du réseau canadien des réserves de biosphère sur leurs pratiques. Même si on y reconnait l'importance de l'éducation et celle des apprentissages collectifs, les acteurs mobilisent peu de théories relatives aux approches, aux modèles et aux stratégies pédagogiques. Ils arrivent peu à justifier leurs pratiques ou à y jeter un regard critique au regard de l'environnement, de l'éducation et de l'ERE.

Par ailleurs, le Secrétariat du Programme MAB a produit des notes techniques pour clarifier la portée de certaines actions suggérées dans la Stratégie de Séville (Bouamrane, 2006, 2007). Un guide en EDD sur les réserves de biosphère a été produit pour la région de la Méditerranée (Scoullos et coll., 2013) ainsi que sur la thématique de la biodiversité (UNESCO, 2017b), permettant de clarifier certaines pratiques éducatives. À cet effet, rappelons que la RBMSH a été l'hôte d'une activité de promotion de l'EDD en 2013. Lors de cet événement, certains acteurs ont exprimé des tensions entre leurs pratiques, l'EDD et certains autres objectifs découlant du Programme MAB. En l'absence d'un modèle cadre offrant des repères aux praticiens, cette réflexion a eu peu d'impact sur les pratiques en ERE ou même en EDD dans la RBMSH.

Signalons enfin que des organisations spécialisées en ERE en France et aux États-Unis ont produit des guides et fiches thématiques (Sardin, Bauer et Thubé, 2008; NAAEE, 2004, 2009, 2017) qui pourraient inspirer le Programme MAB à proposer un modèlecadre en ERE pour les réserves de biosphère, de manière à clarifier, entre autres, les tensions éthiques et politiques entourant les propositions de l'EDD.

\section{Au-delà de l'examen de conformité : vers une évaluation formative des pratiques éducatives}

L'importance de l'évaluation comme processus de rétroaction formatif dans les écrits du Programme MAB est faible. La stratégie de Séville ainsi que le cadre statutaire du Réseau mondial impliquent des examens périodiques tous les 10 ans. Les critères d'examen périodique portant sur les pratiques éducatives sont plutôt quantitatifs et descriptifs. Ils permettent peu de clarifier les pratiques elles-mêmes. Le formulaire d'examen suggère d'identifier les principales institutions d'enseignement actives dans la réserve de biosphère et d'y décrire les programmes destinés aux écoles ainsi qu'aux adultes qui contribuent à la réalisation des objectifs d'une réserve de biosphère. Par ailleurs, on y demande de commenter les changements organisationnels survenus dans 
ces institutions et dans les programmes éducatifs afin d'en apprécier l'efficacité au regard des objectifs de conservation de la biodiversité, de développement durable et de développement des capacités (UNESCO, 2017a).

La pertinence de ces critères d'examen périodique peut être remise en cause à la lumière des observations formulées précédemment, notamment pour dégager un modèle-cadre en ERE propre aux réserves de biosphère ou pour mener une réflexion critique relative aux tensions normatives de l'EDD ainsi qu'aux enjeux éthiques et politiques qui en découlent (Girault et Sauvé, 2008 ; IUCN, 2000 ; Sauvé, 2009). Au-delà d'un exercice de conformité, le caractère formatif de l'examen périodique devrait être renforcé (Reed et Egunyu, 2013) .

\section{Conclusion}

Ce tour d'horizon des écrits du Réseau mondial des réserves de biosphère a permis de mener une réflexion sur les pratiques éducatives proposées. Le recensement des pratiques de la Réserve de biosphère du mont Saint-Hilaire (RBMSH) a permis d'observer la manière dont les acteurs locaux se les approprient. Rappelons que le concept de réserve de biosphère est une forme de reconnaissance qui n'attribue pas de pouvoir d'action. Il est plutôt une invitation à travailler avec la pluralité des acteurs impliqués sur un territoire englobant plusieurs municipalités autour d'une aire protégée centrale.

La diversité des pratiques éducatives de la RBMSH est riche. Elle se traduit principalement par des pratiques «au sujet» de l'environnement et des réserves de biosphère, mais également par des pratiques engagées " pour » la nature. Ces pratiques se déploient surtout dans un cadre non formel. Une réflexion axiologique s'impose dans la RBMSH et le Réseau mondial des réserves de biosphère, notamment pour évaluer la pertinence des pratiques suggérées par l'EDD, ainsi que pour réfléchir aux prescriptions normatives issues des stratégies et plans d'action qui régissent les réserves de biosphère. De quelles représentations de l'environnement, de l'éducation et de l'ERE veut-on faire la promotion pour répondre aux visées des réserves de biosphère?

41 Cette étude de cas démontre certaines tensions entre les pratiques éducatives suggérées à l'échelle internationale et les pratiques à l'échelle locale afin de rendre compte des contextes culturels très variés (Sauvé et coll. 2003) comme celui de la RBMSH. Pour améliorer les pratiques éducatives et les arrimer au contexte local, il serait pertinent de proposer un modèle-cadre plus explicite en éducation relative à l'environnement (Sauvé, 1997a). Cette proposition devrait, entre autres, orienter le processus d'examen périodique de façon à renforcer le caractère formatif de celui-ci (Reed et Egunyu, 2013). Comme le suggèrent Schultz et Lundholm (2010), si les réserves de biosphère visent à devenir des sites d'apprentissage pour améliorer nos rapports à la biosphère, il faudra développer des compétences en évaluation et créer un contexte propice au partage des leçons apprises. 


\section{BIBLIOGRAPHIE}

Bouamrane, M. (2006, Dir.). Biodiversité et acteurs : des itinéraires de concertation Réserves de biosphère.. Notes techniques 1. Paris : UNESCO.

Bouamrane, M. (dir.). (2007). Le dialogue dans les réserves de biosphère : Repères, pratiques et expériences Réserves de biosphère. Notes techniques 2. Paris : UNESCO.

Brianso, I. et Girault, Y. (2014). Innovations et enjeux éthiques des politiques environnementales et patrimoniales : l'UNESCO et le conseil de l'Europe. Éthique publique, 16(1), 1-14.

Bridgewater, P. (2016). The Man and Biosphere program of UNESCO : Rambunctious child of the sixties, but was the promise fulfilled ? Current Opinion in Environmental Sustainability, 19, 1-6.

Buckler, C., et Creech, H. (2014). Façonner l'avenir que nous voulons : Décennie des Nations Unies pour l'éducation au service du développement durable (2005-2014), résumé du rapport final (Rapport No. ED-2014/WS/29). Paris : UNESCO.

Centr'ERE (2018). Mobiliser les acteurs du changement - Stratégie québécoise d'éducation en matière d'environnement et d'écocitoyenneté - Défis, visions et pistes d'action. Montréal : Les Éditions du Centr'ERE.

Centre de la Nature. (2007). Fenêtre sur la Réserve de la biosphère du mont Saint-Hilaire. Musée virtuel du Canada. Repéré 29 décembre 2017, à http://www.museevirtuel.ca/Exhibitions/Hilaire/ html-fr/index.php?page=accueil.html

Centre de la Nature. (2017). Réserve de biosphère de l'UNESCO. Conservation. Centre de la Nature du Mont Saint-Hilaire. Repéré 29 décembre 2017, à http://centrenature.qc.ca/rb-unesco-mab/

EVB-CSQ. (2017). Établissements vert Brundtland - éduquer et agir pour un avenir viable. Centrale des syndicats du Québec (CSQ). Repéré 29 décembre 2017, à http://www.evb.lacsq.org/ fileadmin/user_upload/microsites/eav-evb_internet/documents/Qui_sommes_nous/2017-01__Liste_de_tous_les_EVB__Liste_pour_site_Web_.pdf

FAO. (2010). Stratégie de la FAO en matière de renforcement des capacités (Rapport No. PC 104/3). Rome : L'Organisation pour l'alimentation et l'agriculture (FAO). Repéré à http:// www.fao.org/3/a-k8908f.pdf

Francis, G. (2004). Biosphere reserves in Canada : exploring ideals and experience. Environments, 32(3), 25.

Gagnon, Y.-C. (2012). L'étude de cas comme méthode de recherche. Québec : Presses de l'Université du Québec, 2e édition.

Girault, Y. et Sauvé, L. (2008). L'éducation scientifique, l'éducation à l'environnement et l'éducation pour le développement durable : croisements, enjeux et mouvances. Aster - Revue de recherches en didactique des sciences expérimentales (46), 7-30.

Godmaire, H., Reed, M. et Guertin, M.-A. (2013). Les réserves de biosphère du Canada : apprentissage social et bonnes pratiques. Éducation relative à l'environnement - Regards, Recherche, Réflexions, 11, 233-246.

Godmaire, H., Reed, et M., Potvin, D. (2013). Savoirs partagés : Pratiques exemplaires des réserves de biosphère canadiennes. Ottawa : Commission canadienne pour l'UNESCO. 
Ishwaran, N., Persic, A., et Tri, N. H. (2008). Concept and practice : The case of UNESCO biosphere reserves. International Journal of Environment and Sustainable Development, 7(2), 118.

IUCN. (2000). ESDebate (édité par F. Hesselink, P. P. van Kempen et A. Wals). Union internationale pour la conservation de la nature (UICN), Cambridge.

McGill. (2017). Cours offerts - Une salle de classe en nature. Réserve naturelle Gault de l'Université McGill au mont Saint-Hilaire. Repéré 29 décembre 2017, à https://gault.mcgill.ca/fr/ recherche-et-education/cours-offerts/

MEES (2017). Loi sur l'instruction publique. Gouvernement du Québec : Québec.

MEQ. (2010). Domaines généraux de formation - chapitre 2. Dans Ministère de l'Éducation du Québec (dir.), Programme de formation de l'école québécoise - Enseignement secondaire deuxième cycle (p. 16). Gouvernement du Québec : Québec. Repéré à http:// www.education.gouv.qc.ca/fileadmin/site_web/documents/dpse/formation_jeunes/2pfeq_chap2.pdf

NAAEE. (2004). Guidelines for Excellence Environmental Education Materials. North American Association for Environmental Education, Washington D.C.

NAAEE. (2009). Guidelines for Excellence Nonformal Environmental Education Programs. North American Association for Environmental Education, Washington D.C.

NAAEE. (2017). Guidelines for Excellence Community Engagement. North American Association for Environmental Education, Washington D.C.

Nations Unies. (2008). Le renforcement des capacités-Note pratique. New-York : Programme des Nations-Unies pour le développement. Repéré à https://www.undp.org/content/dam/undp/ library/capacity-development/french/PN \%20Capacity \%20Development_FR_final.pdf

Nations Unies. (2016). Objectif de développement durable - 17 objectifs pour transformer notre monde. Programme de développement durable. Repéré 29 décembre 2017, à http://www.un.org/ sustainabledevelopment/fr/

Price, M. F. (2017). The re-territorialisation of Biosphere Reserves : The case of Wester Ross, Northwest Scotland. Environmental Science and Policy, 72, 30-40.

Raufflet, E., Tengö, M., Guertin, M.-A., Dansou, K. et Filion, L. J. (2010). Landscape entrepreneurship : Lessons from the Mont Saint Hilaire Nature Centre. Dans R. W. Y. Kao (dir.), Sustainable Economy. Corporate, Social and Environmental Responsibility (p. 377-420). London : World Scientific.

Reed, M. G. et Abernethy, P. (2018). Facilitating Co-Production of Transdisciplinary Knowledge for Sustainability : Working with Canadian Biosphere Reserve Practitioners. Society \& Natural Resources, 31(1), 39-56.

Reed, M. G. et Egunyu, F. (2013). Management effectiveness in UNESCO Biosphere Reserves : Learning from Canadian periodic reviews. Environmental Science and Policy, 25, 107-117.

Reed, M. G., Godmaire, H., Abernethy, P. et Guertin, M. A. (2014). Building a community of practice for sustainability : Strengthening learning and collective action of Canadian biosphere reserves through a national partnership. Journal of Environmental Management, 145, 230-0239.

Reed, M. G. et Massie, M. (2013). Embracing ecological learning and social learning : UNESCO biosphere reserves as exemplars of changing conservation practices. Conservation and Society, $11(4), 391$. 
Sandbrook, C., Adams, W. M., Büscher, B. et Vira, B. (2013). Social Research and Biodiversity Conservation. Conservation Biology, 27(6), 1487-1490.

Sardin, J.-P., Bauer, A., \& Thubé, F. (2008). Éducation à l'environnement et la biodiversité (Rapport No. 30). Villiers-en-Bois : Institut de formation et de recherche en éducation à l'environnement en Poitou-Charentes.

Sauvé, L. (1997a). L'approche critique en éducation relative à l'environnement : origines théoriques et applications à la formation des enseignants. Revue des sciences de l'éducation, 23(1), 169.

Sauvé, L. (1997b). Pour une éducation relative à l'environnement - Éléments de design pédagogique, Guide de développement professionnel à l'intention des éducateurs. Montréal : Guérin - Eska, 2e édition

Sauvé, L. (2009). Le rapport entre éthique et politique : un enjeu pour l'éducation relative à l'environnement. Éducation relative à l'environnement - Regards, Recherche, Réflexions, 8 , 147-162.

Sauvé, L., Berryman, T. et Brunelle, R. (2003). Environnement et développement : la culture de la filière ONU. Éducation relative à l'environnement, Regards, Recherches, Réflexions, 4, 33-55.

Scoullos, M., Kouroutos, V., Mantzara, B., Alempei, I. et Malotidi, V. (2013). Education for Sustainable Development in Biosphere Reserves and other Designated Areas - A Resource Book for Educators in South-Eastern Europe and the Mediterranean (UNESCO). Vienne. Repéré à http://unesdoc.unesco.org/images/0021/002199/219946e.pdf

UNESCO. (1996). Réserves de biosphère : la stratégie de Séville et le cadre statutaire du Réseau mondial. Organisation des Nations unies pour l'éducation, la science et la culture (UNESCO), UNESCO - Docs.

UNESCO. (2008). Plan d'action de Madrid (2008-2013). Organisation des Nations unies pour l'éducation, la science et la culture (UNESCO), Paris. Repéré à http://unesdoc.unesco.org/images/ 0016/001633/163301f.pdf

UNESCO. (2016). Plan d'action de Lima pour le Programme sur l'Homme et la biosphère (MAB) de l'UNESCO et son Réseau mondial des Réserves de biosphère (2016-2025). Organisation des Nations unies pour l'éducation, la science et la culture (UNESCO), Paris. Repéré à http://www.unesco.org/ fileadmin/MULTIMEDIA/HQ/sC/pdf/Lima_Action_Plan_fr_final.pdf

UNESCO. (2017a). Le processus d'examen périodique. UNESCO-Sciences naturelles-

Environnement-Sciences écologiques et développement durable-Réserves de biosphère-

Processus d'examen périodique. Organisation des Nations unies pour l'éducation, la science et la culture (UNESCO), Repéré 15 octobre 2017, à http://www.unesco.org/new/fr/natural-sciences/ environment/ecological-sciences/biosphere-reserves/periodic-review-process/

UNESCO. (2017b). Kit pédagogique sur la biodiversité-Volume 1. Organisation des Nations unies pour l'éducation, la science et la culture. 191p.

UNESCO. (2019a). Programme sur l'Homme et la biosphère. UNESCO - Sciences naturelles Environnement - Sciences écologiques et développement durable - Le Programme MAB. Organisation des Nations unies pour l'éducation, la science et la culture (UNESCO), Repéré 29 décembre 2017, à http://www.unesco.org/new/fr/natural-sciences/environment/ecologicalsciences/

UNESCO. (2019b). Réseaux du MAB. UNESCO - Sciences naturelles - Environnement - Sciences écologiques et développement durable - Le Programme MAB - Réseaux du MAB. Organisation des 
Nations unies pour l'éducation, la science et la culture (UNESCO), Repéré 29 décembre 2017, à http://www.unesco.org/new/fr/natural-sciences/environment/ecological-sciences/man-andbiosphere-programme/networks/

UNESCO-PNUE. (1977). Conférence de Tbilissi et Rapport final. Dans Conférence intergouvernementale sur l'éducation relative à l'environnement. Tbilissi (14-26 octobre) : Organisation des Nations unies pour l'éducation, la science et la culture (UNESCO) et Programme des Nations Unies pour l'environnement (PNUE).

Van Cuong, C., Dart, P., et Hockings, M. (2017). Biosphere reserves : Attributes for success. Journal of Environmental Management, 188, 9-17.

\section{NOTES}

1. Le fait que la RBMSH se situe en banlieue d'une métropole comme Montréal a probablement un effet sur les représentations de l'environnement, de l'éducation et de l'éducation relative à l'environnement des acteurs de la réserve. Il serait intéressant de pousser l'analyse des pratiques en ERE au regard de ces considérations géographiques avec d'autres réserves de biosphère périurbaines au Canada ou ailleurs pour apprécier cet effet, identifier les similitudes ainsi que les variations.

2. La portée des examens périodiques des réserves de biosphère est adaptée aux réalités culturelles et organisationnelles de chaque pays membre de l'UNESCO et laissée à la discrétion des Comités nationaux du Programme MAB. L'éducation peut ainsi être plus ou moins considérée lors de tels examens.

3. Il ne s'agit pas d'idéaliser le concept de réserve de biosphère constituée dans le cadre du Programme MAB, mais bien de constater que l'éducation y occupe une place marquée (Ishwaran, et coll., 2008). Suivant l'évolution des pratiques de conservation et de protection de l'environnement, les premières réserves de biosphère, comme celle du mont Saint-Hilaire, ont été créées au début des années 1970, pour la conservation et la recherche, et parfois avec un intérêt mitigé de la part des populations locales. Dans certaines régions du monde, on peut même affirmer que les pratiques de conservation se faisaient contre le gré des populations locales. Cela a affecté leur capacité à relever les défis pour lesquels elles ont été constituées ou ceux qu'épouse actuellement le Programme MAB (Van Cuong, et coll., 2017).

4. Cette analyse a permis d'effectuer un survol des écrits du Programme MAB et d'identifier les travaux de certains auteurs qui ont analysé l'évolution des idées et des pratiques en matière d'ERE et d'EDD au sein des filières de l'ONU et de manière générale en didactique des sciences (Girault et Sauvé, 2008; Sauvé et coll., 2003). Une exploration plus approfondie des écrits de l'UNESCO et du Programme MAB serait certainement intéressante pour apprécier l'évolution des courants en éducation et en ERE, ainsi que l'évolution des pratiques à travers le Réseau mondial des réserves de biosphère.

5. Des critères ont été appliqués au recensement des activités de manière à contribuer au $3 e$ examen périodique de la RBMSH. Les activités et outils recensés abordaient le concept de réserve de biosphère et leurs objectifs (conservation, développement durable, développement des capacités). De plus, ils permettaient d'explorer la diversité des relations « humain-nature » dans l'esprit du Programme MAB. Les activités étaient contextualisées et adaptées au territoire de la RBMSH visant à susciter l'engagement, la participation et le transfert de connaissance ; elles suggéraient des scénarios possibles de résolution de problèmes associés à la RBMSH. De manière pragmatique, les activités ne répondant pas à ces critères n'ont pas été considérées.

6. En raison du caractère non formel des activités, de la période plutôt extensive de l'étude, ainsi que de l'échéancier de l'examen périodique, un portrait quantitatif du recensement n'a pas été 
jugé nécessaire (par exemple: nombre exact d'activités, personnes rejointes, adoption de nouvelles pratiques).

7. L'objectif de l'examen périodique est d'abord de vérifier que la réserve de biosphère réponde bien aux critères de l'article 4 du cadre statutaire. Le bilan proposé par cet examen illustre une tension entre la gestion globale d'un territoire et les pratiques et actions qui en découlent. Le volet éducatif n'échappe pas à cette tension. On peut espérer que la fonction de l'examen évoluera dans le temps. La communauté scientifique et les acteurs des réserves de biosphère sont appelés à enrichir les pratiques des réserves de biosphère de manière générale, y compris en ERE, pour que la gestion du territoire s'améliore à son tour.

\section{RÉSUMÉS}

Le Programme sur l'Homme et la Biosphère (Programme MAB) de l'UNESCO vise à établir une base scientifique pour améliorer les relations entre l'humanité et la nature à l'échelle mondiale. Après presque 50 ans d'existence, l'expression la plus tangible du Programme MAB est un réseau de réserves de biosphère dont l'un des principaux objectifs est l'éducation. Puisque l'éducation relative à l'environnement a pour visée l'harmonisation du réseau de relations personne-sociétéenvironnement, nous avons effectué une analyse critique des actions suggérées à cet effet par le Programme MAB. Cet exercice a été réalisé dans le cadre du $3^{\mathrm{e}}$ examen périodique de la Réserve de biosphère du mont Saint-Hilaire (RBMSH), prévu par le cadre statutaire des réserves de biosphère dans une perspective d'amélioration des pratiques éducatives. Au bilan, nous formulons cinq observations relatives aux visées de l'ERE, au cadre non formel, à l'axiologie des pratiques, à la pertinence d'élaborer un modèle-cadre et à celle d'adopter des approches d'évaluation formative des pratiques pour favoriser la prise en compte de la diversité des relations entre les humains et la biosphère au sein de la RBMSH et dans le Réseau mondial des réserves de biosphère.

UNESCO's Man and the Biosphere (MAB) Program aims to establish a scientific basis for improving human-nature relations on a global scale. After almost 50 years of existence, the most tangible expression of MAB is a network of biosphere reserves, one of whose main objectives is education. Since environmental education (EE) sets out to harmonize the relations between person-society-environment, we have carried out a critical analysis of the actions suggested for this purpose by the MAB. This exercise was carried out as part of the $3^{\text {rd }}$ periodic review of the Mont Saint-Hilaire Biosphere Reserve (RBMSH), in compliance with the Statutory Framework of Biosphere Reserves and with the intention of providing insights into EE practices. In summary, we present five observations concerning the aims of EE, the non-formal framework in which $\mathrm{EE}$ is conducted, the axiology of practices, the relevance of developing an EE framework model, and the adoption of formative evaluation approaches during periodic reviews to take into account the diversity of human-biosphere relations within the RBMSH and the World Network of Biosphere Reserves. 


\section{INDEX}

Keywords : environmental education, education for sustainable development, educational practices, periodic review, biosphere reserve, Man and the Biosphere (MAB), UNESCO, Mont Saint-Hilaire.

Mots-clés : éducation relative à l'environnement, éducation au développement durable, pratiques éducatives, réserve de biosphère, examen périodique, Programme sur l'Homme et la Biosphère (MAB), UNESCO, mont Saint-Hilaire.

Index géographique : Québec

\section{AUTEURS}

\section{MARC-ANDRÉ GUERTIN}

Marc-André Guertin est doctorant en sciences de l'environnement à l'Université du Québec à Montréal (UQAM). Il détient une formation de 2e cycle en éducation relative à l'environnement de l'UQAM et de 3e cycle en pédagogie de l'enseignement supérieur de l'Université de Sherbrooke (UdeS). Il a œuvré au sein du Réseau canadien des réserves de biosphère notamment au Comité canadien du Programme MAB de la Commission canadienne pour l'UNESCO. Il enseigne au Centre universitaire de formation en environnement et développement durable de l'UdeS. (Courriel : Marc-Andre.Guertin@USherbrooke.ca)

\section{GENEVIÈVE POIRIER-GHYS}

Geneviève Poirier-Ghys détient un baccalauréat en biologie de l'Université McGill, une maîtrise en environnement de l'Université de Sherbrooke et un diplôme de 2e cycle en éducation relative à l'environnement de l'UQAM. Elle est responsable de la conservation et des communications pour le Centre de la Nature, l'organisme qui coordonne les activités de la Réserve de biosphère du mont Saint-Hilaire. Elle travaille à promouvoir la conservation des milieux naturels et la participation citoyenne. (Courriel : genevieve@centrenature.qc.ca)

\section{MAUREEN G. REED}

Maureen G. Reed est professeure et directrice adjointe de l'École supérieure d'environnement et de durabilité de l'Université de la Saskatchewan. Elle a mené des recherches sur les réserves de biosphère canadiennes et avec celles-ci depuis 2001 et a été membre du Comité canadien du Programme MAB de la Commission canadienne pour l'UNESCO de 2010 à 2019. (Courriel : maureen.reed@usask.ca)

\section{JÉRÔME DUPRAS}

Jérôme Dupras est professeur au Département des sciences naturelles de l'Université du Québec en Outaouais et chercheur à l'Institut des sciences de la forêt tempérée. Il est titulaire d'un doctorat en géographie et ses intérêts de recherche se centrent sur l'évaluation socioéconomique de la biodiversité et des écosystèmes et l'aménagement du territoire. (Courriel : jerome.dupras@uqo.ca)

\section{GENEVIÈVE RAJOTTE SAURIOL}

Geneviève Rajotte Sauriol détient un baccalauréat en communication de l'UQAM, une maîtrise en environnement de l'Université de Sherbrooke et complète un diplôme de 2e cycle en éducation relative à l'environnement de l'UQAM. Elle est engagée dans diverses causes environnementales 
en plus d'œuvrer comme professionnelle en communication éthique et responsable dans la région de la Mauricie au Québec. (Courriel : genevieve@bleu-foret.ca) 